\title{
Intravitreal aflibercept for neovascular age-related macular degeneration in patients aged 90 years or older: 2-year visual acuity outcomes
}

\author{
Irini Chatziralli $\mathbb{1}^{1} \cdot$ Shane O. Regan ${ }^{2} \cdot$ Ryian Mohamed $\mathbb{1}^{3} \cdot$ James Talks ${ }^{4} \cdot$ \\ Sobha Sivaprasad (10) ${ }^{1}$ UK Aflibercept Users Group
}

Received: 21 October 2017 / Revised: 18 February 2018 / Accepted: 8 April 2018 / Published online: 4 June 2018

(c) The Royal College of Ophthalmologists 2018

\begin{abstract}
Purpose The purpose of this study was to investigate the efficacy of intravitreal aflibercept for neovascular age-related macular degeneration (nAMD) in very elderly patients aged 90 years or older at 2 years after treatment initiation.

Methods In this multicentre retrospective data analysis from electronic medical record, consecutive treatment-naive patients with nAMD treated with aflibercept with at least 2 years follow-up were stratified into those aged $<90$ years (Group I) and an older cohort aged 90 and over (Group II). We compared the visual acuity (EDTRS letters) outcomes at 4 weekly intervals between the two groups over a 2-year period.

Results The mean visual acuity of Group I at presentation was 56.3 ETDRS letters versus 52.8 letters in Group II. Maximal visual acuity was achieved in both the groups by 6 months after initiating treatment (4.7 vs. 4.0 letters gain). By 2 years, the mean visual acuity of the older cohort fell marginally below their baseline visual acuity (0.8 letter loss), while Group I presented +2.1 letters gain. The number of injections given and the retention rate of the older cohort were no different to the rest of the patients.

Conclusions Very old patients with nAMD benefited from aflibercept, but not to the same degree as the younger patients. The study showed that, on an average, the very elderly patients were able to adhere to the intensive anti-VEGF treatment regimens.
\end{abstract}

\section{Introduction}

Age-related macular degeneration (AMD) is broadly classified into early and late forms. Late AMD is the leading cause of visual loss in the elderly. AMD is a perfect

Members of the UK Aflibercept Users Group are listed above References

Sobha Sivaprasad

senswathi@aol.com

1 National Institute for Health Research, Biomedical Research Centre at Moorfields Eye Hospital National Health Service Foundation Trust, London, UK

2 University Hospital Limerick, Limerick, Ireland

3 University College London Hospitals NHS Trust, London, UK

4 Newcastle Upon Tyne Hospitals Foundation NHS Trust, Newcastle-upon-Tyne, UK example of an aging-dependent condition, where the prevalence of the disease increases monotonically with age [1]. The prevalence of late AMD increases from $1 \%$ at 70 years to as high as $12 \%$ in patients over 80 years [2-4]. This condition is an outcome of a multitude of accumulating degenerative changes in the various layers of the outer retina and the choroid. Although there have been many well-conducted epidemiological studies on AMD, the proportions of patients aged 90 years or older in these cohorts were small. Therefore, there is a paucity of information on AMD in the very elderly patients.

Late AMD is further classified into "dry" and "wet or neovascular" (nAMD). The former is responsible for the majority of cases, but there are no treatment options available for this condition as yet. Only $10-15 \%$ of patients exhibit nAMD and these patients present with sudden decrease in vision, and if left untreated, this condition results in profound irreversible visual impairment [5, 6]. AMD is a bilateral disease and the risk of developing nAMD in the fellow eye of patients with unilateral AMD 
increases by $10 \%$ per year from the date of diagnosis, albeit this is dependent on features of the eye and other risk factors [7]. Furthermore, if a patient develops nAMD in one eye at the age of 80 , the risk of nAMD in the fellow eye is $48 \%$ at 10 years [6]. Therefore, we would expect most patients with nAMD at 90 years to have bilateral nAMD. Determining the outcome of treatment in this cohort, compared to those in the younger age group, has implications, as it estimates the burden of this disease in this age group that is also at risk of other aging-dependent co-morbidities. Studying the treatment outcomes in this group will also provide us with better estimates of age-dependent outcomes of treatments for health economic evaluation. Ultimately, this has significant implications for future service provision, and treatment planning in the very elderly.

Intravitreal injections of inhibitors of vascular endothelial growth factor (VEGF) have been the mainstay of treatment for nAMD for over a decade [8-12] The three anti-VEGF agents commonly used for this condition include ranibizumab, bevacizumab and aflibercept. The treatment regimens are intensive with monthly intravitreal injections for 3 months, followed by varying re-treatment regimens depending on the anti-VEGF agent. On an average, a patient requires 7-8 injections in the first year and six injections from the second year. A comprehensive assessment of baseline predictive factors for visual outcomes at month 12 in Comparison of Age-related macular degeneration Treatment Trials (CATT) demonstrated that older age was associated with poor outcomes in both ranibizumab and bevacizumab groups, while the exact cause for this observation remains unknown [10]. Aflibercept is the newest FDA-approved anti-VEGF agent against nAMD, which binds tightly to VEGF-A, having a longer half-life than both ranibizumab and bevacizumab, a property which can be exploited by ophthalmologists to allow greater intervals between intravitreal treatments [12] The VEGF Trap-Eye: Investigation of Efficacy and Safety in Wet AMD studies (VIEW 1 and VIEW 2) showed that intravitreal aflibercept given bimonthly after 3 initial monthly doses had comparable improvement in visual acuity as monthly ranibizumab in treatment-naive patients with nAMD [12]

To our knowledge, the specific effect of older age on the efficacy of aflibercept in nAMD has not been addressed in the scientific literature, while older age has been shown to adversely affect the outcomes in patients treated with both bevacizumab and ranibizumab $[10,11]$ In addition, since it is difficult to study the outcomes in an elderly population due to several issues, including follow-up retention, large study samples are needed in order to reach reliable conclusions. Consequently, analysis of a large database system seems to be a good option. In fact, the use of electronic databases to estimate the outcomes in retinal disorders is becoming increasingly common, and they have been used to provide estimates for national and regional population outcomes [13, 14]. We have recently utilized a national dataset from 16 centres in the United Kingdom (UK) to report that the "real world" clinical outcomes of aflibercept in nAMD are similar to that of the randomized control trials of aflibercept in AMD [13] Therefore, this dataset is ideal to examine the influence of advanced age on visual outcomes in patients with nAMD treated with aflibercept. In this study, we compared aflibercept the outcomes in very elderly patients with nAMD ( $\geq 90$ years) with the rest of the cohort treated with aflibercept for nAMD to evaluate the treatment's outcomes in the very old population.

\section{Materials and methods}

In this retrospective study, anonymised data was extracted from 16 UK National Health Service (NHS) hospitals forall treatment-naive eyes with nAMD initiated on aflibercept between March 2013 and April 2015, to ensure a minimum of 24-month follow-up. All the data were recorded using a single electronic medical record (EMR) system (Medisoft Ophthalmology; Medisoft Limited, Leeds, UK), which mandated the collection of a standardized dataset throughout the nAMD care pathway. Written approval for anonymised data extraction was received from each participating hospital. Ethical approval was not required, since a study of this nature is considered as an audit or service evaluation. The study was conducted in accordance with the tenets of Helsinki Declaration and the UK's Data Protection Act.

Sites were selected based on the confirmation that they used aflibercept to treat nAMD according to the VIEW protocol, consisting of 3-monthly intravitreal aflibercept injections (loading phase), followed by repeated bimonthly injections thereafter during theentire year 1 . All included centers were using a treat and extend approach after year 1 . To determine the influence of age on visual acuity (VA) outcomes, patients were divided into two groups: Group I consisted of a younger cohort aged 0-89 years, while Group II consisted of very elderly patients aged 90 years and over.

All patients underwent optical coherence tomography (OCT) and fluorescein angiography to confirm the diagnosis of nAMD, while indocyanine green angiography was used at the discretion of the physician when needed. Eyes with polypoidal choroidal vasculopathy, choroidal neovascularization of other cause than nAMD, other co-morbidities that may confound visual outcomes and those treated previously with anti-VEGF were excluded. At each visit, visual acuity, injection history and follow-up date were recorded and entered into the EMR system by all staff members as part of routine clinical care. Potential operative and postoperative ocular and systemic complications were also documented at each visit. 
A

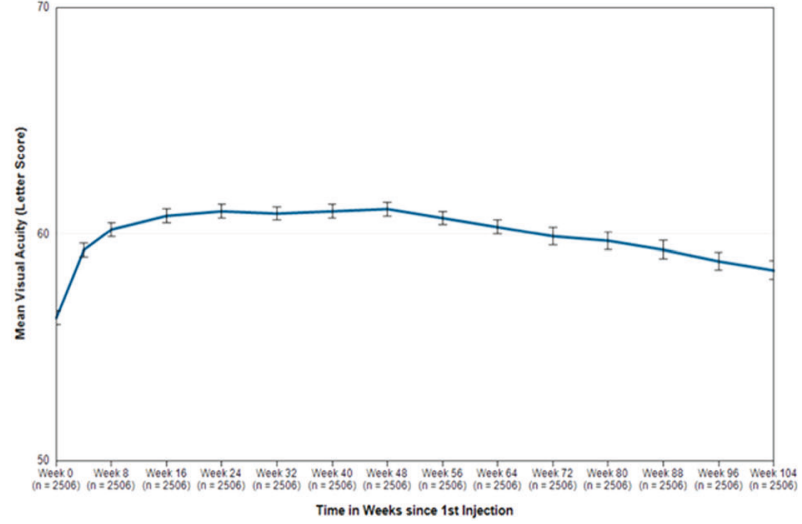

B

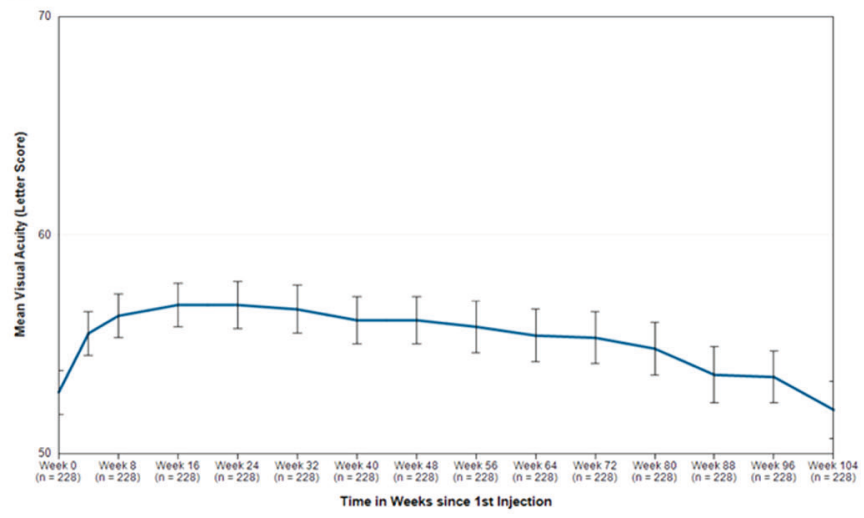

Fig. 1 Mean change in visual acuity over time in patients aged $<90$ years (a) and $\geq 90$ years (b)

\section{Visual acuity and missing value imputation}

Early Treatment Diabetic Retinopathy Study (ETDRS) visual acuity letter scores at 2 meters were recorded at each visit at all sites, and represented the primary outcome of the study. In cases when alternatives measures were used conversion to ETDRS letters was performed. The visual acuity at each clinical visit was used for the analysis. When visual acuity recorded was counting figures, hand movements, light perception or no light perception, zero ETDRS letter score was ascribed.

Since in real-world studies, patients tend not to attend at the precisely intended intervals, the visual acuity of all attendees at 4-week intervals were utilized for the analysis over a 2-year period, having ensured that the sample size in each month reflected all patients who were being followed up continually. If there were gaps in the visual acuity data, they were imputed using the mean of the observations before and after the missing period. No observations were carried forward beyond the last recorded VA value, because loss to follow-up is more common in clinical practice than in proper clinical trials.

\section{Results}

Data were extracted from 16 NHS centers for 2734 treatment-naive eyes initiating aflibercept treatment for nAMD between March 2013 and April 2015, to allow a minimum follow-up of 2 years. Group I (aged $<90$ years) comprised 2506 eyes and Group II (aged $\geq 90$ years) included 228 (8.3\%) eyes.

\section{Mean change in visual acuity at 2 years}

The mean change in VA over time for both the groups is shown in Fig. 1. In patients aged $<90$ years, the mean VA at baseline was 56.3 ETDRS letters (standard error, 0.3 letter), improving significantly to 60.9 ETDRS letter at year 1 (standard error, 0.3 letter; +4.6 letters gain) and to 58.4 ETDRS letter at year 2 (standard error, 0.4 letter; +2.1 letters gain), compared to baseline. In patients aged $\geq 90$ years, the VA at baseline was 52.8 ETDRS letters (standard error, 1 letter) and improved to 55.9 ETDRS letters at year 1 (standard error, 1.2 letter; +3.1 letters gain), but dropped to 52 ETDRS letters at year 2 (standard error, 1.3 letter; -0.8 letters loss), compared to baseline. The maximum change in VA was achieved at 6 months for both the groups $(+4.7$ and +4.0 letters gain respectively).

\section{First eye involvement}

If analysis was restricted to first-treated eyes (eyes with normal vision in the other eye), in Group I the mean baseline VA for first-treated eyes was 55.2 ETDRS letters (standard error, 0.4 letter), which increased to 60.6 letters at year 1 (standard error, 0.4 letter; +5.4 letters gain) and to 58.1 letters (standard error, 0.5 letter; +5 letters gain) at year 2. In Group II, the mean baseline VA in first-treated eyes was 50.0 ETDRS letters (standard error, 1.2 letter), increasing to 52.4 letters at year 1 (standard error, 1.5 letter; +2.4 letters gain), but dropping to 48.8 letters at year 2 (standard error, 1.7 letter; -1.2 letters loss).

\section{Second eye involvement}

For second-treated eye (eyes with visual loss in other eye) in Group I, the mean baseline VA was 63.5 ETDRS letters (standard error, 0.7 letter), increasing to 65.1 letters at year 1 (standard error, 0.8 letter; +1.6 letters gain) and to 63.4 letters at year 2 (standard error, 0.8 letter; -0.1 letter loss). In Group II, the mean baseline VA for second-treated eye was 59.2 ETDRS letters (standard error, 2.4 letter), which increased to 63.0 letters at year 1 (standard error, 2.2 letter; 

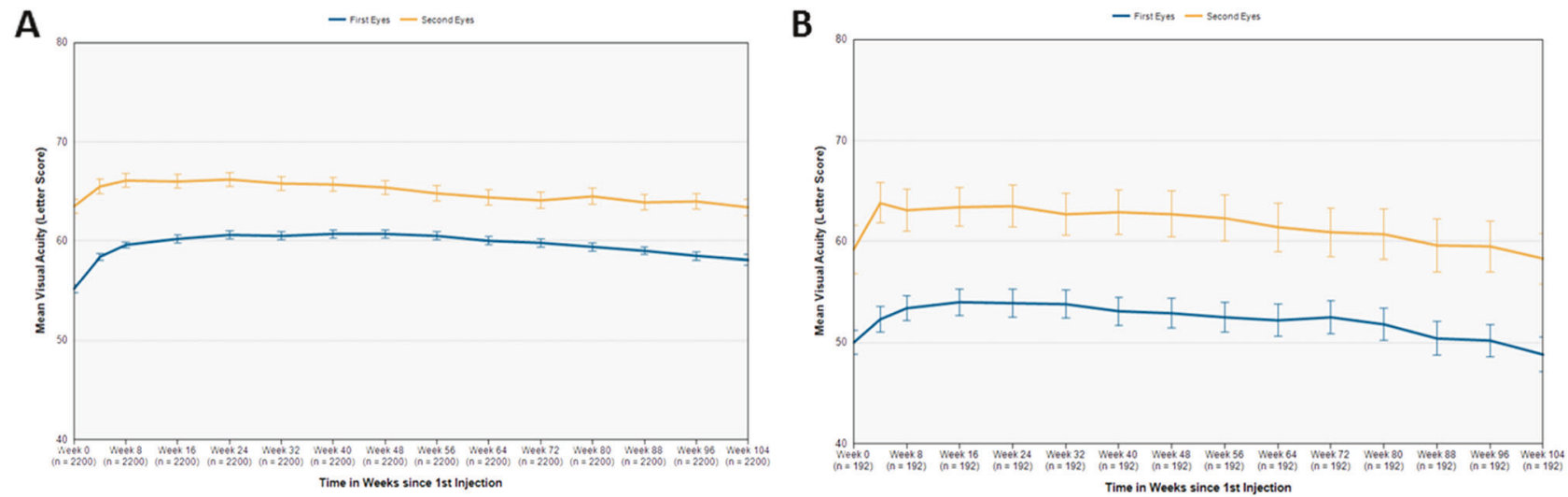

Fig. 2 Mean change in visual acuity over time regarding first-treated and second-treated eyes in patients aged $<90$ years (a) and $\geq 90$ years $(\mathbf{b})$

+3.8 letters gain) and dropped to 58.3 letters at year 2 (standard error, 2.5 letter; -0.9 letter loss), as it is shown in Fig. 2.

\section{Patients presenting poor visual acuity}

There were 218 eyes $(13.0 \%)$ in Group I, compared to 24 eyes $(10.5 \%)$ in Group 2 that presented with $\mathrm{VA}<35$ ETDRS letters. For both the groups, the largest VA gains were achieved in eyes that started with the worst vision. In patients aged $<90$ years, eyes with baseline VA $<35$ ETDRS letters gained a mean of 11.6 letters (standard error, 1.1 letter) and 9.2 letters (standard error, 1.2 letter) at year 1 and 2 respectively. A similar trend was observed in patients aged $\geq 90$ years with eyes commencing aflibercept treatment, with the worst VA attaining the largest VA gains, but to a lesser degree. Specifically, patients in Group II with baseline VA $<35$ ETDRS letters gained a mean of 7.4 letters (standard error, 1.9 letter) and 3.9 letters (standard error, 3.4 letter) at year 1 and 2, respectively.

\section{Patients presenting good vision}

There were 397 patients (15.8\%) in Group I, compared to 24 (10.5\%) patients in Group II who presented VA > 70 ETDRS letters. In Group I, there was a mean decline of 0.6 letters (standard error, 0.4 letter) and 3.5 letters (standard error, 0.6 letter) at year 1 and 2, respectively, as it is depicted in Fig. 3a. In Group II, patients with baseline VA $>70$ ETDRS letters (24 eyes) experienced a significant drop in VA of 3.8 letters at 1 year (standard error, 1.7 letter) and 13.3 letters at year 2 (standard error, 3.4 letter), as shown in Fig. $3 b$.

\section{Gains in visual acuity}

In Group I, the proportions of the 2147 eyes (85.7\%) with follow-up at year 1 that gained 5, 10 and 15 letters were
$52.3 \%, 35.2 \%$ and $20.6 \%$, respectively. At year 2, the proportions of the 2102 eyes $(83.9 \%)$ with a gain of 5,10 and 15 letters were $46.7 \%, 31.5 \%$ and $20.7 \%$, respectively. In patients aged $\geq 90$ years, the proportions of 186 eyes $(81.6 \%)$ with follow-up at 1 year that gained 5, 10 and 15 letters were $49.5 \%, 32.8 \%$ and $22.6 \%$, respectively. At year 2 , the proportions of the 192 eyes $(84.2 \%)$ gaining 5, 10 and 15 letters were $37.5 \%, 27.6 \%$ and $17.7 \%$, respectively (Fig. 4).

\section{Losers in visual acuity}

Over the first year, the proportions of those losing 5, 10 and 15 letters in Group I were $18.8 \%, 10 \%$ and $5.8 \%$, respectively and at 2 years, $26.3 \%, 18.3 \%$ and $13 \%$ lost 5,10 and 15 letters. In Group II, the proportions losing 5, 10 and 15 letters were $26 \%, 14 \%$ and $7 \%$, respectively at year 1 and $37 \%, 24 \%$ and $16 \%$ of eyes in year 2 , respectively.

\section{Discussion}

This study evaluated aflibercept outcomes in NAMD in the largest series of patients aged 90 years or over. The principal message of this study is that intravitreal aflibercept is effective in the very elderly population with nAMD and they are able to adhere to rigorous anti-VEGF treatment regimens.

There are half a million people aged 90 years or older in the UK. Owen et al reported $\sim 40,000$ new nAMD cases a year in the UK, with an estimated 7700 people beingin this age group (19\%) [4]. This study shows that the oldest people constituted $8 \%$ of the new cases presented, and were treated with aflibercept over 2 years. Therefore, approximately half the expected number of the very elderly patients with new onset nAMD were present for the treatment. Considering those who presented for diagnosis and 

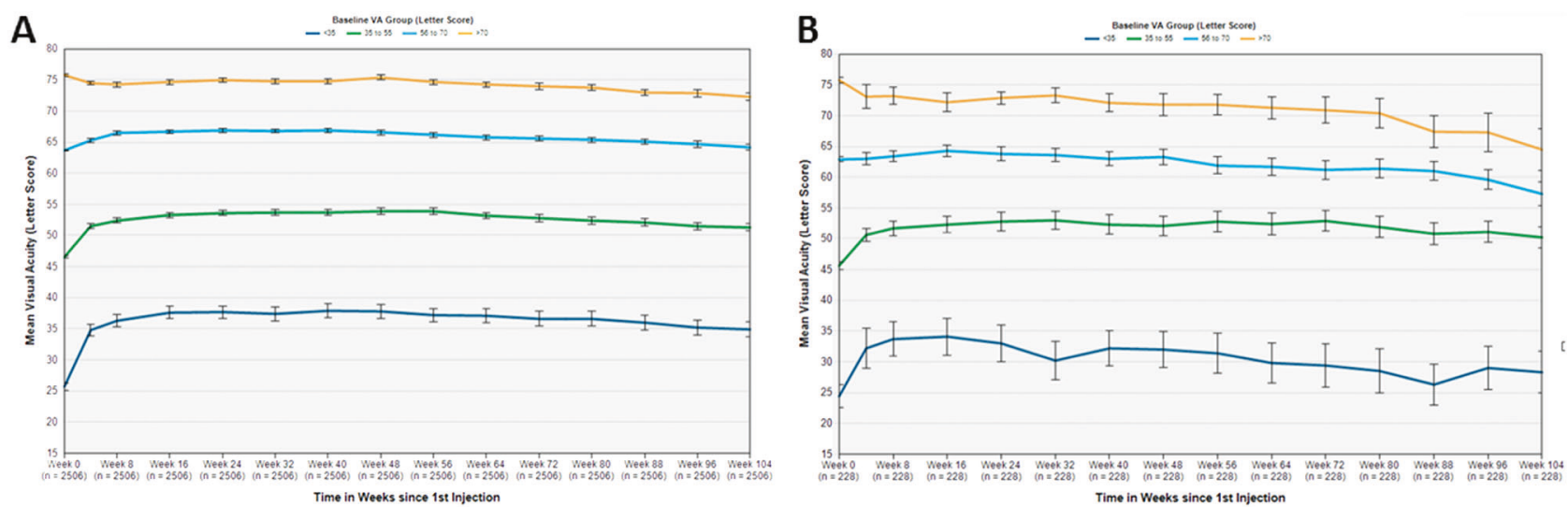

Fig. 3 Mean change in visual acuity over time based on baseline visual acuity groups in patients aged $<90$ years (a) and $\geq 90$ years (b)

treatment, their baseline VA was similar to the rest of the cohort. Therefore, those were the patients who were able to access the NHS benefit from the treatment received for nAMD.

The proportion of patients with final VA records at 2 years was also similar between the two the groups, suggesting that the drop out rate was not higher in the very old patients. The study is limited to 2-year follow-up. Longer follow-up of these patients will provide insight into any deviations between the two cohorts on clinic attendance with time. The outcome measured in various ways show that very elderly patients with nAMD benefit from aflibercept therapy. However, it is more challenging for the patients aged 90 years or above to achieve the driving vision, defined as more than 70 letters in the treated eyes. In addition, the older patients who presented good vision $(>70$ letters) showed a larger decline in VA over the 2 years, compared to the rest of the cohort. These outcomes in this age group may contribute to the higher risk of falls and fractures, need for nursing home support and mortality.

Interestingly, we also observed that $80 \%$ of patients presented first eye involvement in the 90 years and above group. We postulate that this group may indeed represent a unique "special" group that has avoided conversion to nAMD, probably having a lower predisposition for the development of nAMD. It would be useful to interrogate this group of patients further in terms of both functional and structural changes in the retina to study the disease mechanism.

The VA outcomes of the present study were not as favorable as the clinical trials outcomes, but were similar to the other real-life studies $[13,15,16]$ The mean gain in VA in our study in patients aged $<90$ years was +4.6 and +2.1 letters at year 1 and 2, respectively. In patients aged $\geq 90$ years, the mean gain in VA at year 1 was +3.1 letters, while patients lost 0.8 letters at year 2 . The integrated analysis of VIEW 1 and VIEW 2 studies showed 8.4 letters gain at year 1 (7.9 letters for VIEW 1 and 8.9 letters for VIEW 2 study [12], whereas Talks et al found 5.1 letters gain at year 1 in patients with nAMD treated with intravitreal aflibercept in real-life conditions, based on the VIEW protocol [13]. Additionally, previous audits using PRN ranibizumab in clinical practice reported VA gains of 2-3.8 letters at year 1 $[15,16]$. It is worthy to note that reduced VA in the older group may represent more prevalent concurrent atrophic changes than in the younger group. In addition, the aged outer retinal tissue may be less responsive to anti-VEGF therapy.

The discrepancy in the VA outcomes between clinical trials and real-life studies may be explained by the fact that in the real-world, data measurement techniques may differ. Specifically, VA is usually measured with the patients' habitual correction and not with the subjective refraction at each visit as in clinical trials. Although this may underestimate the actual changes in vision, it may reflect better patients' vision experience [13]. Moreover, patients in clinical trials are strictly selected and may have different characteristics than those of daily practice, while treatment is administered within tight timelines. It is also worthy to note that this older patient cohort is often not included in the clinical trials, even though these diseases are aging dependent. They represented $8 \%$ of this cohort. Therefore, this is an example where the answers to a research question are better derived from real-life datasets than clinical trials.

Another interesting observation of our study was that maximal VA gain was obtained at month 6 after initiation of treatment, irrespective of the age. In addition, the initial VA gain achieved after the 3-monthly loading phase was maintained at the end of year 1 in both the groups and by year 2 in patients aged $<90$ years. In contrast, in the very elderly patients, the rate of decline in VA was more pronounced after year 1, with patients presenting loss of 0.8 letters at year 2 and falling below the baseline VA. However, the outcome is still better than the natural history of the disease. Although treatment after 6 months did not result in further VA gains in either group, continuous treatment may halt further visual deteriorations in both the groups. 

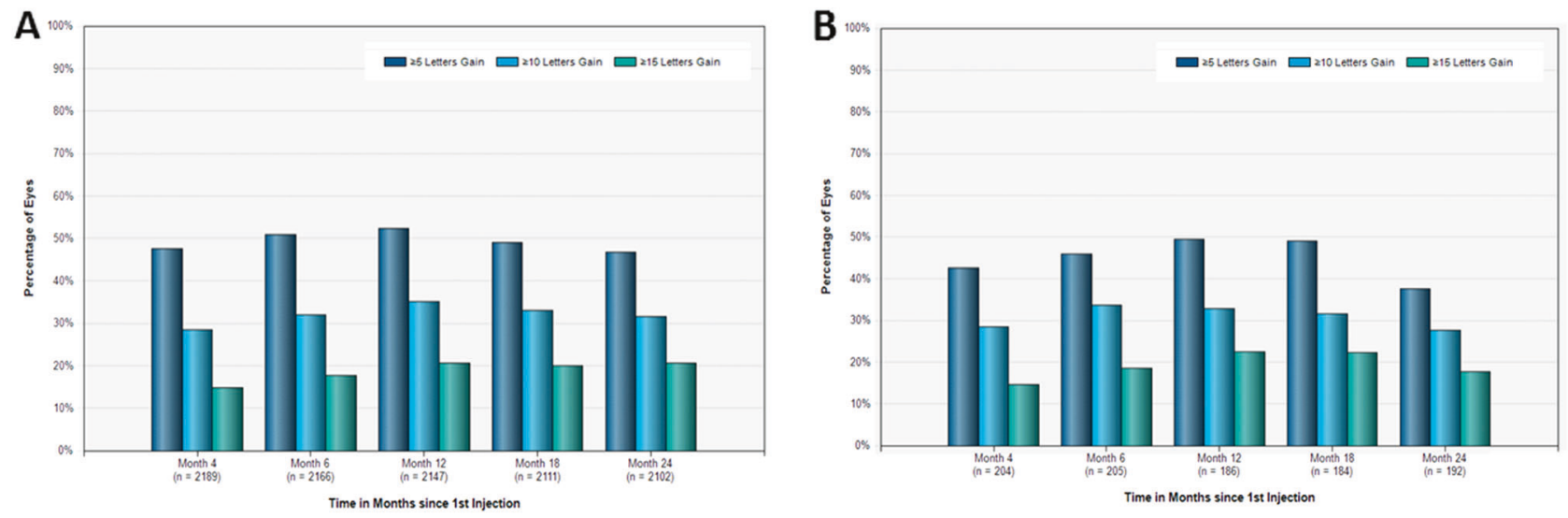

Fig. 4 Percentage of patients gaining at least 5, 10 and 15 letters in patients aged $<90$ years (a) and $\geq 90$ years (b)

In conclusion, our study showed that very old patients with nAMD benefited from aflibercept therapy, but not to the same degree as the younger patients. The reason why elderly patients do not achieve the same outcomes as their younger counterparts despite adhering to the treatment regimen is unclear. An understanding of the morphological differences in patients with nAMD across different age profiles should shed more light on the disease mechanisms. The information provided in this study may also be of value to health economists for incorporation in future costeffectiveness models.

\section{Summary}

\section{What was known before}

- Intravitreal aflibercept is safe and effective for the treatment of age-related macular degeneration.

- The so-far studies regarding the effectiveness of aflibercept in patients over 90 years are scarce.

\section{What this study adds}

- Very old patients with nAMD benefited from aflibercept, but not to the same degree as younger the patients.

- The study showed that on an average, the very elderly patients are able to adhere to the intensive anti-VEGF treatment regimens.

UK Aflibercept Users Group A. J. Lotery, F. Ghanchi, N. Patel, C. Bailey, S. Mahmood, A. Lobo, B. Paul, Q. Kashif, C. Santiago, G. Walters, M. Tahir, B. Mushtaq, K. Ahmed, M. McKibbin, S. Sivaprasad, and J. Talks

\section{Compliance with ethical standards}

Conflict of interest Irini Chatziralli, James Talks and Sobha Sivaprasad have received travel grants, research grants and speaker fees, and attended advisory board meetings of Bayer, Allergan and Novartis. The remaining authors declare that they have no conflict of interest.

\section{References}

1. Myall DJ, Pitcher TL, Pearson JF, Dalrymple-Alford JC, Anderson TJ, MacAskill MR. Parkinson's in the oldest old: Impact on estimates of future disease burden. Park Relat Disord. 2017. https://doi.org/10.1016/j.parkreldis.2017.06.018.

2. Colijn JM, Buitendijk GHS, Prokofyeva E, Alves D, Cachulo ML, Khawaja AP, et al. Prevalence of age-related macular degeneration in europe: the past and the future. Ophthalmology . 2017. https://doi.org/10.1016/j.ophtha.2017.05.035

3. Bunce C, Xing W, Wormald R. Causes of blind and partial sight certifications in England and Wales: April 2007-March 2008. Eye .2010;24:1692-9.

4. Owen CG, Jarrar Z, Wormald R, Cook DG, Fletcher AE, Rudnicka AR. The estimated prevalence and incidence of late stage age related macular degeneration in the UK. Br J Ophthalmol. 2012;96:752-6.

5. Ferris FL 3rd, Wilkinson CP, Bird A, Chakravarthy U, Chew E, Csaky K, et al. Clinical classification of age-related macular degeneration. Ophthalmology . 2013;120:844-51.

6. Chew EY, Clemons TE, Agrón E, Sperduto RD, Sangiovanni JP, Davis MD, et al. Ten-year follow-up of age-related macular degeneration in the age-related eye disease study: AREDS report no. 36. JAMA Ophthalmol. 2014;132:272-7.

7. Clemons TE, Milton RC, Klein R, Seddon JM, Ferris FL 3rd. ; Age-Related Eye Disease Study Research Group. Risk factors for the incidence of Advanced Age-Related Macular Degeneration in the Age-Related Eye Disease Study (AREDS) AREDS report. Ophthalmology. 2005;112:533-9.

8. Rosenfeld PJ, Brown DM, Heier JS, Boyer DS, Kaiser PK, Chung $\mathrm{CY}$, et al. Ranibizumab for neovascular age-related macular degeneration. N Engl J Med. 2006;355:1419-31.

9. Brown DM, Kaiser PK, Michels M, Soubrane G, Heier JS, Kim $\mathrm{RY}$, et al. Ranibizumab versus verteporfin for neovascular agerelated macular degeneration. N Engl J Med. 2006;355:1432-44.

10. Martin DF, Maguire MG, Ying GS, Grunwald JE, Fine SL, Jaffe GJ. Ranibizumab and bevacizumab for neovascular age-related macular degeneration. N Engl J Med. 2011;364:1897-908.

11. Chakravarthy U, Harding SP, Rogers CA, Downes SM, Lotery AJ, Culliford LA, et al. Alternative treatments to inhibit VEGF in age-related choroidal neovascularisation: 2-year findings of the IVAN randomised controlled trial. Lancet . 2013;382:1258-67. 
12. Heier JS, Brown DM, Chong V, Korobelnik JF, Kaiser PK, Nguyen QD, et al. Intravitreal aflibercept (VEGF trap-eye) in wet age-related macular degeneration. Ophthalmology. 2012;119: 2537-48.

13. Talks JS, Lotery AJ, Ghanchi F, Sivaprasad S, Johnston RL, Patel N, et al. First-year visual acuity outcomes of providing aflibercept according to the VIEW Study Protocol for age-related macular degeneration. Ophthalmology . 2016;123:337-43.

14. Barthelmes D, Nguyen V, Daien V, Campain A, Walton R, Guymer R, et al. Two year outcomes of "treat and extend" intravitreal therapy using aflibercept preferentially for neovascular age-related macular degeneration. Retina . 2017. https://doi.org/ 10.1097/IAE.0000000000001496.

15. Pushpoth S, Sykakis E, Merchant K, Browning AC, Gupta R, Talks SJ. Measuring the benefit of 4 years of intravitreal ranibizumab treatment for neovascular age-related macular degeneration. Br J Ophthalmol. 2012;96:1469-73.

16. Holz FG, Tadayoni R, Beatty S, Berger A, Cereda MG, Cortez R, et al. Multi-country real-life experience of anti-vascular endothelial growth factor therapy for wet age-related macular degeneration. Br J Ophthalmol. 2015;99:220-6. 